\title{
An analytical model for the growth of quantum dots on ultrathin substrates
}

\author{
S. P. A. Gill ${ }^{\text {a) }}$ \\ Department of Engineering, University of Leicester, University Road, Leicester LE1 7RH, United Kingdom
}

(Received 2 March 2011; accepted 7 April 2011; published online 22 April 2011)

The self-assembly of heteroepitaxial quantum dots on ultrathin substrates is analyzed within the context of small perturbation theory. Analytical expressions are derived for the dependence of the quantum dot separation on the substrate thickness. It is shown that the substrate thickness is critical in determining this separation when it is below the intrinsic material length scale of the system. The model is extended to simultaneous dot growth on both sides of the substrate. It is shown that vertically anticorrelated structures are preferred with an increase in the dot separation of $15 \%$ above that found in the one-sided case. (c) 2011 American Institute of Physics. [doi:10.1063/1.3583447]

The instability of elastically strained heteroepitaxial thin films leads to the self-assembly of quantum dot (QD) nanostructures. Known as the Asaro-Tiller-Grinfeld (ATG) instability, ${ }^{1}$ the size and spacing of the QDs is determined by the competition between the energetic driving forces (strain and surface energy) and the kinetics of the material transport process (surface diffusion). Recently, it has been shown that some control of the QD size and spacing can be achieved through varying the thickness of the substrate. ${ }^{2,3}$ The energetics of the system are strongly affected when the thickness of the substrate is comparable with the QD length scale. For ultrathin substrates, such as nanomembranes, ${ }^{2,4}$ significant stress relief can arise due to bending of the substrate. The local strain field beneath the QDs is also modified due to the proximity of the lower free surface. If QDs are simultaneously deposited on both sides they can interact through the substrate to create a vertically anticorrelated QD structure. ${ }^{2}$ In this paper the ATG instability model is extended to consider growth of QDs on ultrathin substrates to quantify how the substrate thickness affects the QD size and spacing and their spatial correlation.

The problem is formulated within the context of a kinetic variational principle, ${ }^{5}$ whereby

$$
\Pi=\Psi+\dot{G},
$$

has contributions from the dissipation potential, $\Psi$, which represents the work done in material transport, and the rate of change in the Gibb's free energy of the system, $\dot{G}$, which provides the driving force for the evolution. The optimal kinetic field is that which render the variational functional stationary, $\delta \Pi=0$.

First we consider a single planar epitaxial film of thickness $h_{0}$ (see Ref. 6 for morphological effects). This is subject to a perturbation of amplitude $A(t)$ and wavelength $\lambda$, such that $h(x, t)=h_{0}+A(t) \sin (2 \pi x / \lambda)$, as shown in Fig. 1. Mass conservation requires that $\left[d\left(j_{s}\right) / d x\right]+v_{n}=0$, where $j_{s}$ is the material surface flux and the normal velocity of the surface $v_{n} \approx \dot{h}$ for small slopes $(A \ll \lambda)$. The dissipation potential (per unit wavelength) is then

\footnotetext{
${ }^{a)}$ Electronic mail: spg3@1e.ac.uk.
}

$$
\Psi=\int_{0}^{\lambda} \frac{j_{s}^{2}}{2 D_{s}} d x=\frac{\lambda^{3}}{16 \pi^{2} D_{s}} \dot{A}^{2}
$$

where $D_{s}$ is the surface diffusion coefficient.

The Gibb's free energy has surface energy and elastic strain energy contributions. For an isotropic surface energy density, $\gamma_{0}$, and a surface elastic strain energy density, $w(x)$, one has ${ }^{5}$

$$
\dot{G}=\int_{0}^{\lambda}\left(\gamma_{0} \kappa+w\right) v_{n} d x
$$

where $\kappa(x) \approx\left(d^{2} h / d x^{2}\right)$ is the surface curvature.

The elasticity problem consists of two parts: global bending/stretching of the substrate by the initially planar film; and a local sinusoidal contribution from the thin film perturbation, as shown in Fig. 1. These are solved separately and combined through linear superposition. The film in Fig. 1 is subject to a (compressive) mismatch strain $\epsilon_{m}<0$ which is relaxed by bending of the assembly. Let the thickness of the substrate be $2 c$ and assume the variation in the normal strain in the $x$-direction through the thickness is linear such

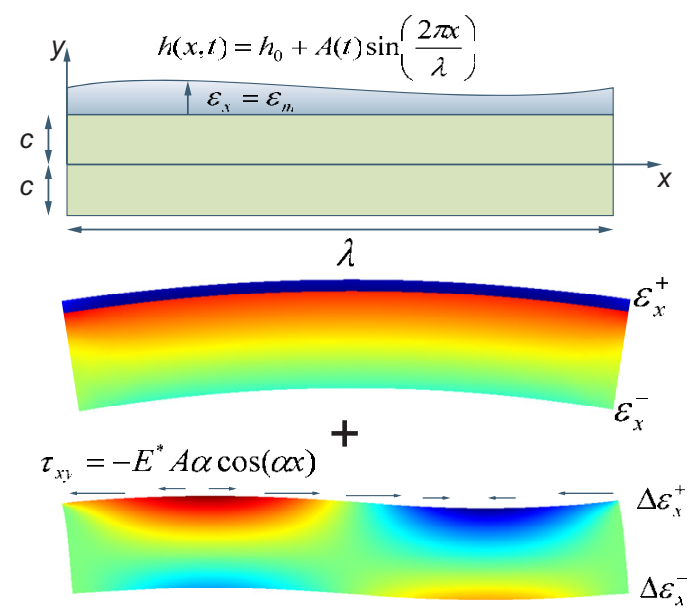

FIG. 1. (Color online) Geometry of the film-substrate assembly (top). The epitaxial film experiences a mismatch strain $\epsilon_{m}$ and a sinusoidal surface perturbation of amplitude $A$ and wavelength $\lambda$. The assembly can relieve the strain by a combination of global elongation and bending (middle) with the addition of a sinusoidal component due to the local surface waviness (bottom). Contours show magnitude of stress $\sigma_{x}$ in the substrate. 
that $\epsilon_{x}(y)=\epsilon_{0}+K y$, where $K$ is the (elastic bending) curvature of the assembly and $\epsilon_{0}$ is a uniform elongation. The total elastic strain energy (per wavelength) is

$$
\begin{aligned}
W= & \frac{1}{2} \int_{-c}^{h_{0}+c} \sigma_{x} \epsilon_{x} d y=\frac{E^{*}}{2} \int_{-c}^{c} \epsilon_{x}^{2} d y \\
& +\frac{E^{*}}{2} \int_{c}^{h_{0}+c}\left(\epsilon_{m}+\epsilon_{x}\right)^{2} d y,
\end{aligned}
$$

where the normal stress in the $x$-direction, $\sigma_{x}=E^{*} \epsilon_{x}$, and $E^{*}$ $=\left[E /\left(1-\nu^{2}\right)\right]$ is given by the Young's modulus $E$ and Poisson's ratio $\nu$ of the substrate and film (assumed to be the same). This is minimized when

$$
K=-\frac{3 \eta \epsilon_{m}}{c(1+\eta)^{3}} \quad \epsilon_{0}=-\frac{\left(\eta^{2}-\eta+1\right) \eta \epsilon_{m}}{(1+\eta)^{3}},
$$

where $\eta=h_{0} / 2 c$ is the film-to-substrate thickness ratio.

The total strain on the top surface of the film is now $\epsilon_{x}^{+}+\Delta \epsilon_{x}^{+}$, where $\epsilon_{x}^{+}=\epsilon_{x}\left(h_{0}+c\right)=\left\{\epsilon_{m}\left[1-2 \eta-(1 / 2) \eta^{2}\right] /(1\right.$ $\left.+\eta)^{3}\right\}$ is the uniform strain due to extension/bending and $\Delta \epsilon_{x}$ is the perturbation to the strain field due to the waviness in the film profile. This latter contribution can be modeled for small slopes as a distributed surface traction, whereby the surface shear stress component $\tau_{x y}=-E^{*} \epsilon_{x}^{+}(d h / d x){ }^{7}$ The internal stresses must satisfy the standard biharmonic equation ${ }^{8}$

$$
\frac{\partial^{4} \phi}{\partial x^{4}}+2 \frac{\partial^{4} \phi}{\partial x^{2} \partial y^{2}}+\frac{\partial^{4} \phi}{\partial y^{4}}=0,
$$

where $\sigma_{x}=\partial^{2} \phi / \partial y^{2}, \quad \sigma_{y}=\partial^{2} \phi / \partial x^{2}$ and the shear stress $\tau_{x y}=-\partial^{2} \phi / \partial x \partial y$. The solution is

$$
\phi=\sin (\alpha x)\left[\left(C_{1}+y C_{3}\right) \cosh \alpha y+\left(C_{2}+y C_{4}\right) \sinh \alpha y\right],
$$

where $\alpha=2 \pi / \lambda$. The constants $C_{i}$ are chosen to satisfy the boundary conditions $\sigma_{y}=0$ and $\tau_{x y}=-E^{*} \epsilon_{x}^{+} A \alpha \cos (\alpha x)$ on the top surface (at $y=c^{\prime}$ ) and $\sigma_{y}=0$ and $\tau_{x y}=0$ on the bottom surface (at $y=-c^{\prime}$ ), where $c^{\prime}=c+(1 / 2) h$ is the half-thickness for this assembly. Hence the strain perturbations on the upper $(+)$ and lower $(-)$ surfaces are

$$
\Delta \epsilon_{x}^{ \pm}=-\epsilon_{x}^{+} A \alpha\left(g_{1} \pm g_{2}\right) \sin (\alpha x),
$$

where the functions

$$
g_{1}(\xi)=\frac{\cosh ^{2} \xi}{\left(\frac{1}{2} \sinh 2 \xi+\xi\right)} \quad g_{2}(\xi)=\frac{\sinh ^{2} \xi}{\left(\frac{1}{2} \sinh 2 \xi-\xi\right)},
$$

with $\xi=2 \pi c^{\prime} / \lambda$, define the substrate thickness effect in relation to the dot spacing, $\lambda$. These functions are shown in Fig. 2 . For ultrathin substrates $(\xi \ll 1), g_{i} \rightarrow B_{i} / \xi$, where $B_{1}=1 / 2$ and $B_{2}=3 / 2$. For thicker substrates $(\xi \gg 1), g_{i} \rightarrow 1$ and the ATG result ${ }^{5}$ is recovered as required.

The total strain energy density on the surface is $w(x)$ $=(1 / 2)\left(\sigma_{x}^{+}+\Delta \sigma_{x}^{+}\right)\left(\epsilon_{x}^{+}+\Delta \epsilon_{x}^{+}\right) \approx E^{*} \epsilon_{x}^{+} \Delta \epsilon_{x}^{+}+w_{0}^{+}$for small perturbations, where $w_{0}^{+}=(1 / 2) E^{*}\left(\epsilon_{x}^{+}\right)^{2}=w_{0} f(\eta)$ is the constant strain energy on the film surface, $w_{0}=(1 / 2) E^{*} \epsilon_{m}^{2}$ is the strain energy in a film on a thick substrate and $f(\eta)=[1-2 \eta$ $\left.-(1 / 2) \eta^{2}\right]^{2} /(1+\eta)^{6}$. Hence

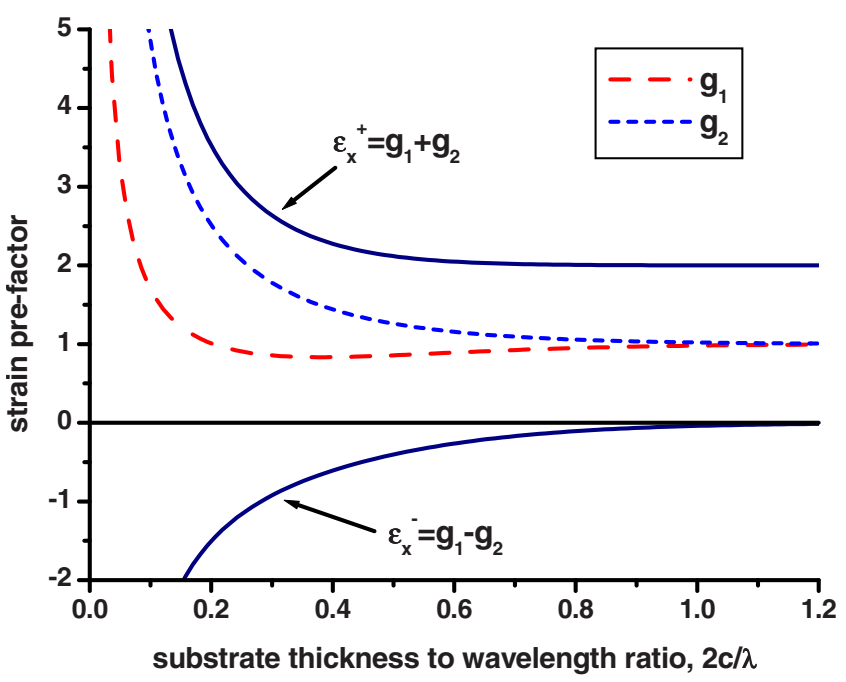

FIG. 2. (Color online) The two functions, $g_{1}$ and $g_{2}$, define the surface strain perturbation (8) and depend on the substrate thickness relative to the dot separation, $\xi$.

$$
w(x)=w_{0}^{+}[1-2 A \alpha g \sin (\alpha x)],
$$

where $g(\xi)=g_{1}+g_{2}$, and the rate of change in Gibbs free energy (3) is

$$
\dot{G}=-2 \pi\left[w(\eta, \xi)-\frac{\pi \gamma_{0}}{\lambda}\right] A \dot{A},
$$

where the strain energy $w(\eta, \lambda)=w_{0} f(\eta) g(\xi)$ accounts for both the global bending/extension of the substrate, $f(\eta)$, and the local sinusoidal deformation field due to the film perturbation, $g(\xi)$.

The variational functional (1) is defined by (2) and (11). The growth rate that minimizes this functional is $\dot{A}=\beta A$, where

$$
\beta=\frac{16 \pi^{3} D_{s}}{\lambda^{4}}\left[w(\eta, \xi) \lambda-\pi \gamma_{0}\right] .
$$

The observed (fastest growing) wavelength will be that which maximizes $\beta$. This stationary value occurs when

$$
\frac{d g}{d \xi}+\frac{3 g}{\xi}=\frac{2 \gamma_{0}}{c^{\prime} w_{0} f(\eta)} \text {. }
$$

This has no simple solution but the wavelength can be approximated in the thin and ultrathin regimes as

$$
\begin{aligned}
& \frac{\lambda}{\lambda_{0}}=\frac{1}{f(\eta)} \text { for } \mathrm{c} \gg \frac{\lambda}{2 \pi}, \\
& \frac{\lambda}{\lambda_{0}}=\sqrt{\frac{12 \pi(1+\eta)}{\operatorname{Bf}(\eta)} \frac{\mathrm{c}}{\lambda_{0}}} \quad \text { for } \mathrm{c} \ll \frac{\lambda}{2 \pi},
\end{aligned}
$$

where $B=2\left(B_{1}+B_{2}\right)=4$ and $\lambda_{0}=2 \pi \gamma_{0} / 3 w_{0}$ is the ATG (thick substrate) wavelength. The range of validity of these approximations can be seen in Fig. 3. It suggests that taking the smaller of the two wavelengths gives a reasonable rough estimate. It can be seen that the wavelength becomes increasingly dependent on the substrate thickness as its thickness $c$ decreases below $\lambda_{0} / 2 \pi f(\eta)$. Such a decrease in the QD spacing with decreasing substrate thickness has been observed by Ritz et al. ${ }^{2}$ for Ge QDs on 6 and $23 \mathrm{~nm}$ Si nanomembranes. 


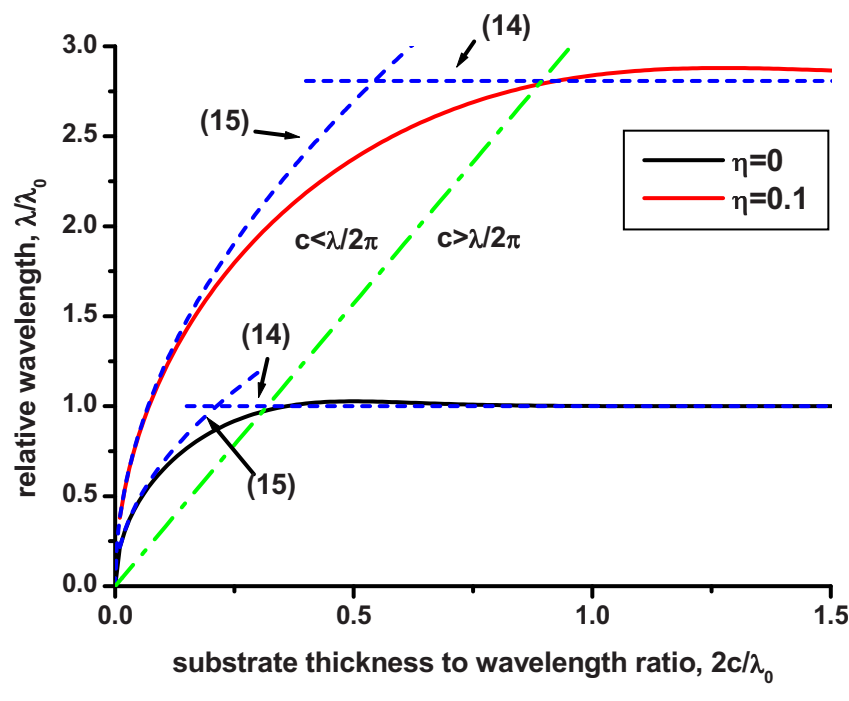

FIG. 3. (Color online) The relative wavelength (QD spacing) as a function of the relative substrate thickness for two different relative thicknesses of epitaxial film ( $\eta=0$ and 0.1$)$.

Substitution in Eq. (12) gives the optimal growth rate

$$
\begin{aligned}
& \frac{\beta}{\beta_{0}}=f(\eta)^{4} \quad \text { for } \mathrm{c} \gg \frac{\lambda}{2 \pi}, \\
& \frac{\beta}{\beta_{0}}=\left[\frac{\mathrm{Bf}(\eta)}{12 \pi(1+\eta)} \frac{\lambda_{0}}{\mathrm{c}}\right]^{2} \text { for } \mathrm{c} \ll \frac{\lambda}{2 \pi},
\end{aligned}
$$

where $\beta_{0}=81 D_{s} w_{0}^{4} / \gamma_{0}^{3}$ is the classic ATG growth rate.

The analysis is now extended to consider simultaneous growth of QDs on both sides of the substrate. Introduce a film on the lower $(-)$ surface with a surface profile $h(x, t)$ $=h_{0}+A(t) \sin (\alpha x+\delta)$, where $\delta$ is the phase difference between the top and bottom growth modes. The strain perturbation on the top surface $(+)$ is the superposition of the two fields, (8), so for the two-sided case

$$
\Delta \boldsymbol{\epsilon}_{x}^{+}=-\boldsymbol{\epsilon}_{x}^{+} A \alpha\left[\left(g_{1}+g_{2}\right) \sin (\alpha x)+\left(g_{1}-g_{2}\right) \sin (\alpha x+\delta)\right],
$$

The rest of the analysis proceeds as before. There is no bending of the assembly (due to symmetry) and hence $f(\eta)$ $=1 /(1+2 \eta)$ from pure extension. The strain field interactions give $B=B_{1}(1+\cos \delta)+B_{2}(1-\cos \delta)$. The expected growth mode is the one that maximizes the growth rate (17) and hence maximize $B$. The minimum value of $B=1$ occurs when the dots are vertically correlated $(\delta=0)$. The maximum value
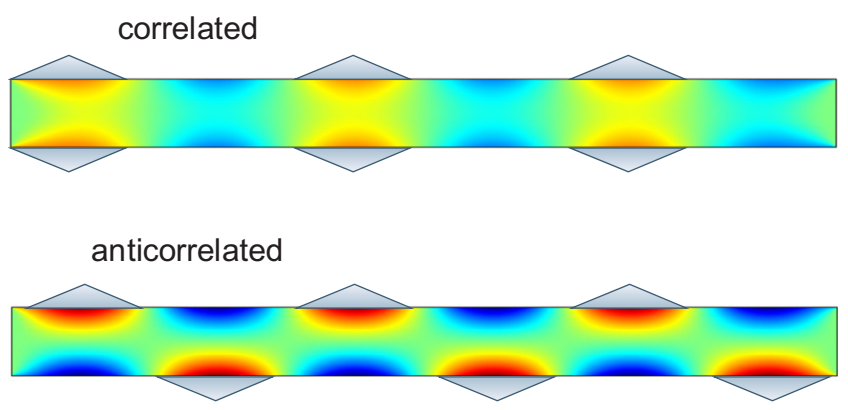

FIG. 4. (Color online) Vertically anticorrelated QD growth is strongly preferred over correlated growth. Contours show magnitude of stress $\sigma_{x}$ in the substrate.

of $B=3$ occurs when the dots are anticorrelated $(\delta=\pi)$. These two scenarios are shown in Fig. 4. Hence it is always expected that dots will be vertically anticorrelated when they can influence each other through the ultrathin substrate. This corresponds with experimental observations. ${ }^{2}$ It is predicted that the wavelength for two-sided growth will be $2 / \sqrt{3}$ $=1.15$ times the value in the single-sided case.

In conclusion, an analytical model for the stability of epitaxial films on an ultrathin substrate has been presented. The substrate thickness effect is incapsulated in the functions $f(\eta)$ (stress relief due to bending/extension) and $g(\xi)$ (stress change due to surface modulation), where $\eta=h_{0} / 2 c$ is the ratio of film thickness $\left(h_{0}\right)$ to substrate thickness $(2 c)$ and $\xi=2 \pi c^{\prime} / \lambda$ depends on the ratio of the total thickness $\left(c^{\prime}\right)$ to the QD separation $(\lambda)$. It is found that the substrate thickness is critical in determining this wavelength for $c<\lambda / 2 \pi$. Furthermore, it is shown that vertically anticorrelated QD structures are preferred for two-sided growth and that the dot separation will be $15 \%$ greater than in the single-sided case.

${ }^{1}$ R. J. Asaro and W. A. Tiller, Metall. Trans. 3, 1789 (1972); M. A. Grinfeld, J. Nonlinear Sci. 3, 35 (1993).

${ }^{2}$ M. Huang, C. S. Ritz, B. Novakovic, D. Yu, Y. Zhang, F. Flack, D. E. Savage, P. G. Evans, I. Knezevic, F. Liu, and M. G. Lagally, ACS Nano 3, 721 (2009); C. S. Ritz, H.-J. Kim-Lee, D. M. Detert, M. M. Kelly, F. S. Flack, D. E. Savage, Z. Cai, P. G. Evans, K. T. Turner, and M. G. Lagally, New J. Phys. 12, 103011 (2010).

${ }^{3}$ Y. Ni and L. H. Ho, Appl. Phys. Lett. 97, 261911 (2010).

${ }^{4}$ H.-J. Kim-Lee, D. E. Savage, C. S. Ritz, M. G. Lagally, and K. T. Turner, Phys. Rev. Lett. 102, 226103 (2009).

${ }^{5}$ S. P. A. Gill and T. Wang, Surf. Sci. 602, 3560 (2008).

${ }^{6}$ H. Wang, Y. Zhang, and F. Liu, J. Appl. Phys. 104, 054301 (2008); H. Hu, H. J. Gao, and F. Liu, Phys. Rev. Lett. 101, 216102 (2008).

${ }^{7}$ S. P. A. Gill, Appl. Phys. Lett. 89, 203115 (2006).

${ }^{8}$ S. P. Timoshenko and J. N. Goodier, Theory of Elasticity, 3rd ed. (McGraw-Hill International, 1970), p. 53. 